\title{
Trombosis aguda arterial y venosa en pacientes infectados con COVID-19
}

\section{Acute arterial and venous thrombosis in patients infected with COVID-19}

\author{
Ana K. Trujillo-Araujo*, Alfonso Cossío-Zazueta, Víctor M. Camarillo-Nava, y Roberto C. Serrato-Auld \\ Servicio de Angiología y Cirugía Vascular, Hospital de Especialidades “Antonio Fraga Mouret”, Centro Médico Nacional La Raza, Instituto Mexicano \\ del Seguro Social (IMSS), Ciudad de México, México
}

\begin{abstract}
Resumen
Antecedentes: Los pacientes infectados por COVID-19 sufren complicaciones y los procesos trombóticos se relacionan de forma estrecha con la infección. Objetivo: Describir los factores vinculados con aumento del riesgo de episodios trombóticos en la COVID-19. Material y métodos: Estudio de casos y controles para identificar factores relacionados con los procesos trombóticos en pacientes con COVID-19. Se utilizó estadística descriptiva, comparativa e inferencial, así como regresión logística. Se determinó un valor de $p<0.05$ como estadísticamente significativo. Se empleó el programa estadístico IBM SPSS V.25. Resultados: Se atendieron 15 casos de trombosis agudas, el $17.5 \%$ arteriales y el $8.8 \%$ venosos; el $66.7 \%$ $(n=10)$ correspondió a hombres. Se usó anticoagulación terapéutica y se practicaron procedimientos quirúrgicos. Se identificaron como factores de riesgo la presentación de alguna comorbilidad $(p=0.005)$ y el TTPa prolongado $(p=0.010)$, así como alteraciones en el dímero $D$. Conclusiones: Los pacientes infectados sufren hipercoagulabilidad y ésta les confiere un alto riesgo de trombosis. La elevación significativa de dímero $D$ puede ser uno de los marcadores de inflamación. La COVID-19 no es contraindicación para algún procedimiento de revascularización, lo que debe llevar a mejorar la conducta diagnóstica y terapéutica en pacientes con infección por COVID-19.
\end{abstract}

Palabras clave: COVID-19. Trombosis arterial. Trombosis venosa. Dímero D.

\section{Abstract}

Background: There are recognized complications in patients infected by COVID-19 and thrombotic processes are closely related to infection. Objective: To describe the factors associated with increased risk in thrombotic events in COVID-19. Material and methods: Case-control study to identify factors associated with thrombotic processes in patients with COVID-19. Descriptive, comparative and inferential statistics were used, as well as logistic regression. The value of $p<0.05$ was determined as statistically significant and the statistical program IBM SPSS V.25 was used. Results: 15 cases of acute thrombosis were assesed, $17.5 \%$ were arterial and $8.8 \%$ were venous, $66.7 \%(n=10)$ were men. Therapeutic anticoagulation and surgical procedures were neccesary. There was an association as a risk factor the fact of presenting some comorbidity $(p=0.005)$, and prolonged aPTT ( $p=0.10)$, as well as abnormalities in the D-dimer. Conclusions: Infected patients suffers from hypercoagulability and confers a high risk of thrombosis. Significant elevation of D-dimer may be one of the markers of inflammation. The condition of presenting COVID-19 is not a contraindication for any revascularization procedure, which encourages us to improve our diagnostic and therapeutic behavior in patients with COVID-19 infection.

Key words: COVID-19. Arterial thrombosis. Venous thrombosis. D-dimer.

\section{Correspondencia:}

*Ana K. Trujillo-Araujo

E-mail: anatru0208@gmail.com
Disponible en internet: 23-06-2021

Rev Mex Angiol. 2021;49(2):45-50 www.RMAngiologia.com

0377-4740/@ 2021 Sociedad Mexicana de Angiología y Cirugía Vascular y Endovascular, A.C. Publicado por Permanyer. Este es un artículo open access bajo la licencia CC BY-NC-ND license (http://creativecommons.org/licenses/by-nc-nd/4.0/). 


\section{Introducción}

La enfermedad por coronavirus del 2019 (COVID-19) se debe a un virus relacionado con el síndrome respiratorio agudo grave por coronavirus 2 (SARS-CoV-2) 1 . Alrededor del $20 \%$ de los pacientes muestra complicaciones, incluidos los trastornos de la coagulación. Existe cierto consenso general de que el proceso inflamatorio que se desarrolla después de una infección viral se desencadena por una activación masiva de macrófagos que generan una "tormenta de citocinas"2,3. La evidencia emergente sugiere que las formas graves y críticas de la COVID-19 están mediadas, en parte, por un estado de hipercoagulabilidad caracterizado por angiopatía trombótica microvascular y macrovascular ${ }^{4-6}$. La prevalencia de trombosis entre pacientes con $\mathrm{CO}$ VID-19 no se ha establecido con precisión, ya que la mayor parte de las publicaciones médicas se centra en pacientes hospitalizados que tienen más probabilidades de tener afecciones comórbidas que las personas con carga de enfermedad leve 0 asintomáticas ${ }^{6}$.

EI SARS-CoV-2 es un coronavirus de RNA monocatenario, que ingresa a las células humanas sobre todo al unirse a la enzima convertidora de angiotensina 2 $(\mathrm{ACE} 2)^{7}$. Las anomalías de laboratorio comunes que se encuentran en pacientes con COVID-19 incluyen linfopenia y elevación de la lactato deshidrogenasa y marcadores inflamatorios como la proteína $C$ reactiva, el dímero D, la ferritina y la interleucina-6 (IL-6) ${ }^{6}$. Las anomalías hemostáticas más constantes con la COVID-19 incluyen trombocitopenia leve y mayores valores del dímero $\mathrm{D}(>1 \mathrm{mg} / \mathrm{ml})$ que se relacionan con un resultado leta| $\left.\right|^{8,9}$. Se ha observado también una desregulación de la vía de la urocinasa durante la infección del coronavirus que produce un mayor daño pulmonar $^{10}$. En individuos normales, las plaquetas contribuyen con más de la mitad de la fuerza total del coágulo, pero en los pacientes con COVID-19, la fuerza está dominada por el componente de fibrina ${ }^{11}$. La activación excesiva del complemento puede provocar microangiopatía trombótica difusa (MAT) y disfunción de órganos terminales ${ }^{12}$.

La trombosis arterial (TA) se desarrolla en el $4.4 \%$ de los pacientes con COVID-19 grave ${ }^{4}$. La evidencia emergente sugiere que la COVID-19 se vincula con endotelitis, caracterizada en términos histológicos por daño endotelial difuso e infiltración por células inflamatorias ${ }^{12,13}$. El inicio de la presentación clínica de la trombosis no siempre inicia con los síntomas por la infección de coronavirus y se presenta incluso hasta 19 días después (promedio, 11-23) ${ }^{3}$. El tromboembolismo arterial en la población general afecta con más frecuencia a hombres en comparación con las mujeres ${ }^{14,15}$. Las infecciones virales empeoran las afecciones vasculares preexistentes, además de inducir directamente lesiones miocárdicas y vasculares ${ }^{16}$. Los pacientes con COVID-19 también tienen un mayor riesgo de tromboembolismo venoso (TEV). Además de la inmovilización prolongada, el daño endotelial y la inflamación vascular contribuyen al desarrollo de un estado de hipercoagulabilidad ${ }^{17}$. Existe una creciente evidencia de que existe una endotelitis inducida por virus y esto puede contribuir a la hipercoagulación ${ }^{18}$. En ausencia de datos de alta calidad, la profilaxis farmacológica debe reservarse para los pacientes con mayor riesgo, incluidos aquellos con movilidad limitada y antecedentes de TEV o neoplasia maligna activa ${ }^{19,20}$. Las observaciones realizadas hasta ahora con pacientes con COVID-19 en todo el mundo llevan a la probable conclusión de que los pacientes con diabetes e hipertensión como comorbilidad tienen un mayor riesgo de experimentar efectos adversos graves debido a la infección, incluidos los trombóticos ${ }^{21}$. Este artículo intenta describir los factores vinculados con aumento del riesgo de episodios trombóticos en la COVID-19, así como el tratamiento óptimo para los que ya los tienen.

\section{Material y métodos}

Existen factores de riesgo que elevan el riesgo de desarrollar complicaciones trombóticas agudas relacionadas con SARS-CoV-2.

\section{Objetivo}

Describir los factores vinculados con aumento del riesgo de episodios trombóticos en COVID-19. Se realizó un estudio de casos y controles en los expedientes de pacientes con y sin alguna manifestación trombótica aguda vascular atendidos en el Hospital de Especialidades Dr. Antonio Fraga Mouret, del Centro Médico Nacional (HECMN) La Raza durante el periodo de marzo a agosto de 2020. Tras obtener la autorización del comité de investigación se obtuvieron los datos de todos los pacientes con el diagnóstico clínico de trombosis aguda (trombosis arterial: pérdida repentina de la perfusión de las extremidades hasta dos semanas después del episodio inicial/trombosis venosa: formación de un trombo en una o más de las venas profundas, por lo general en las piernas) de los expedientes clínicos y estudios de imagen (tomografía con ventana 
pulmonar de alta resolución, TACAR) en este periodo en el departamento de archivo clínico, en el sistema institucional de optimización de camas y sistema de cómputo de estudios radiológicos de esta unidad médica.

\section{Criterios de inclusión para casos}

Pacientes con prueba confirmatoria de reacción en cadena de la polimerasa (PCR) o TACAR con CORADS de 4 o 5 que presentaran alguna manifestación trombótica aguda arterial o venosa, que contaran con expediente clínico completo y fueran mayores de 18 años, hospitalizados y valorados en el HECMN La Raza.

\section{Criterios de exclusión para casos}

Pacientes que tuvieran secuela postrombótica o antecedente de enfermedad arterial periférica y recibieran tratamiento para este.

\section{Criterios de inclusión para controles}

Pacientes que tuvieran infección por SARS-CoV-2 que contaran con expediente clínico completo, mayores de 18 años, hospitalizados y valorados en el HECMN La Raza, sin diagnóstico de trombosis venosa profunda o insuficiencia arterial aguda.

\section{Análisis estadístico}

Las variables cualitativas se resumieron con frecuencias y porcentajes, y las cuantitativas con media y desviación estándar. Para conocer el tipo de distribución se utilizaron las pruebas de bondad y ajuste de Kolmogorov-Smirnov. Los resultados de las variables continuas se expresaron como media \pm desviación estándar (DE) y aquellos con una distribución asimétrica como mediana y rango; para las variables categóricas se emplearon frecuencia absoluta y relativa. Se realizaron comparaciones de las variables entre los grupos y se analizaron con $\chi^{2}$ (variables categóricas) y una prueba $U$ de Mann-Whitney (variables continuas). Se determinaron razón de momios (RM) entre desenlace y cada variable independiente, y su intervalo de confianza al 95\%, y se utilizó regresión logística. En todos los casos se determinó una $p \leq 0.05$ como estadísticamente significativa. Se empleó el programa Excel de Windows 10 y el paquete estadístico IBM SPSS V. 25.
Tabla 1. Datos generales de los participantes del estudio

\begin{tabular}{|l|c|c|}
\hline & $\mathbf{n =} \mathbf{5 7}$ & $\%$ \\
\hline Sexo & & \\
$\quad$ Masculino & 32 & 56.1 \\
Femenino & 25 & 43.9 \\
\hline $\begin{array}{l}\text { Comorbilidad } \\
\text { Ninguna }\end{array}$ & & \\
Diabetes mellitus & 21 & 36.8 \\
Hipertensión arterial & 11 & 19.3 \\
Otras & 6 & 10.5 \\
Más de 2 comorbilidades & 6 & 10.5 \\
\hline & 13 & 22.8 \\
\hline Edad & $\boldsymbol{H}$ & $\mathbf{D E}$ \\
\hline IMC & 57 & 13.9 \\
\hline
\end{tabular}

$\mathrm{n}$ : frecuencia; \%: porcentaje; $\mu$ : media; DE: desviación estándar.

Tabla 2. Frecuencia de episodios trombóticos, tipos de trombosis y tratamiento en pacientes hospitalizados por COVID-19 en el HECMN La Raza

\begin{tabular}{|l|c|c|}
\hline & $\mathbf{n}$ & $\%$ \\
\hline Trombosis & & \\
\hline Presente & 15 & 26.3 \\
\hline Ausente & 42 & 73.7 \\
\hline $\begin{array}{l}\text { Tipo de trombosis } \\
\quad \text { Arterial }\end{array}$ & 10 & 17.5 \\
$\quad$ Venosa & 5 & 8.8 \\
\hline Tratamiento & & \\
\hline Médico & & 14 \\
\hline Quirúrgico & 8 & 12.3 \\
\hline
\end{tabular}

\section{Resultados}

Se revisó un total de 182 expedientes, 57 con criterios para incluirlos en este estudio; 15 pacientes (26.3\%) se identificaron con trombosis aguda y 42 pacientes fueron los controles. De los expedientes estudiados, el $56 \%$ correspondió al sexo masculino ( $n=32$ ). El $36.8 \%$ de los participantes no mostró ninguna comorbilidad, pero entre quienes la sufrieron la más frecuente fue la diabetes mellitus con $19.3 \%$ ( $n=$ 11). El promedio de edad de los participantes fue de 57 años ( \pm 13.9 ), el menor de 21 y el mayor de 86; el IMC fue de 28.5 ( \pm 5.42 ) con intervalo de 19.4 a 47.9 (los datos se detallan en la Tabla 1). Se atendieron 15 casos de trombosis aguda, de los cuales el $17.5 \%$ correspondió a trombosis agudas arteriales $(n=10)$ y el $8.8 \%$ a venosas $(n=5)$, con $66.7 \%(n=10)$ del sexo masculino y el $33.3 \%(n=5)$ del femenino (Tabla 2). 
Tabla 3. Datos bioquímicos entre pacientes hospitalizados con COVID-19 con y sin trombosis en el HECMN La Raza

\begin{tabular}{|l|c|c|c|c|c|c|c|}
\hline \multirow{2}{*}{} & \multicolumn{2}{|c|}{ Total } & \multicolumn{2}{c|}{ Casos } & \multicolumn{2}{c|}{ Control } \\
\cline { 2 - 8 } & Mediana & Intervalo & H & DE & H & DE \\
\hline DHL (U/L) & 402 & $52-2006$ & 383 & $159-1864$ & 403 & $52-2,006$ & 0.593 \\
\hline TTP $(s)$ & 31.7 & $21-144$ & 38.6 & $21-144$ & 30.7 & $22-60$ & 0.046 \\
\hline PLAQ $(\mathrm{k} / \mu \mathrm{L})$ & 220 & $15-545$ & 226 & $58-546$ & 209 & $15-524$ & 0.878 \\
\hline Dímero D $(\mathrm{mg} / \mathrm{L})$ & $0.50^{*}$ & $0.12-13.8$ & .62 & $0.30-8.99$ & 0.43 & $0.12-13.80$ & 0.041 \\
\hline PCR $(\mathrm{mg} / \mathrm{L})$ & 90 & $1.5-462$ & 75 & $1.51-315$ & 95 & $5-462$ & 0.163 \\
\hline
\end{tabular}

* $=U$ de Mann-Whitney.

Tabla 4. Factores de riesgo entre pacientes hospitalizados con COVID-19 con y sin trombosis en el HECMN La Raza

\begin{tabular}{|l|c|c|c|c|c|}
\hline \multirow{2}{*}{ Sexo } & \multirow{2}{*}{ OR } & \multicolumn{2}{|c|}{ IC95\% } & \multirow{2}{*}{$\chi^{2}$} & p \\
\cline { 3 - 5 } & & Mín & Máx & & \\
\hline Diabetes & 1.16 & 0.85 & 1.5 & 0.916 & 0.339 \\
\hline Hipertensión & 0.943 & 0.389 & 2.284 & 0.017 & 0.897 \\
\hline $\begin{array}{l}\text { Presencia de alguna } \\
\text { enfermedad }\end{array}$ & 0.718 & 0.303 & 1.703 & 0.559 & 0.454 \\
\hline Obesidad & 1.575 & 20.171 & 7.782 & 0.005 \\
\hline Edad >65 a & 0.755 & 0.45 & 1.2 & 1.7 & 0.189 \\
\hline Dímero D & 1.258 & 0.516 & 3.069 & 0.259 & 0.611 \\
\hline Plaquetas & 1.49 & 0.98 & 2.28 & 3.61 & 0.057 \\
\hline PCR & 0.90 & 0.66 & 1.2 & 0.443 & 0.506 \\
\hline TTPa & 1.463 & 0.613 & 3.488 & 0.742 & 0.389 \\
\hline
\end{tabular}

*Presencia de más de dos comorbilidades, incluidas diabetes mellitus e

hipertensión, cardiopatía, enfermedad reumatológica, enfermedad renal o hepática

El 53\% ( $n=8)$ ingresó a hospitalización por síntomas respiratorios y durante su estancia se reconoció afectación vascular y se solicitó la valoración del servicio de angiología; hasta $13 \%(n=2)$ acudió por síntomas vasculares, pero estos pasaron a segundo término por los signos de dificultad respiratoria; en el resto $33 \%$ $(n=5)$, la valoración inicial se efectuó por los síntomas vasculares y se estableció el diagnóstico de infección por COVID durante su hospitalización. Debido a las condiciones observadas durante la contingencia, se determinó solo el diagnóstico de trombosis mediante hallazgos clínicos e interrogatorio (directo o indirecto). El tratamiento se dividió en médico y quirúrgico: ocho pacientes con el primero y siete con el segundo (los datos se hallan en la Tabla 2). El tratamiento médico se basó en heparina de bajo peso molecular (enoxaparina), que se ajustó por peso si no existía contraindicación 0 , en caso contrario, se ajustó para pacientes renales; los tipos de procedimientos practicados fueron los siguientes: trombectomía femoral arterial $(n=1)$, trombectomía femoral arterial + amputación supracondílea ipsolateral ( $n=1)$, amputación supracondílea $(n=4)$ e infracondílea $(n=1)$, trombectomía humeral arterial $(n=2)$, trombectomía femoral y poplítea arterial con derivación femoropoplítea con injerto PTFEe $(n=1)$. Dentro de las complicaciones no se agregaron amputaciones posteriores al tratamiento de revascularización; el $13.3 \%$ de todos los pacientes tratados sufrió claudicación (una de las trombosis arteriales que solo recibieron tratamiento médico y una exploración femoral). En cuanto a los datos bioquímicos, la mediana de DHL en el grupo de los casos fue de 383, a diferencia de los controles que fue de 403. El TTP tuvo una mediana de $38.6 \mathrm{~s}$ en los casos en comparación con $30.7 \mathrm{~s}$ en el grupo de los controles. Para el dímero D (mg/L) se presentó una mediana de 0.62 en los casos respecto de 0.43 en el grupo control; se observaron diferencias estadísticamente significativas en cuanto al TTP y el dímero $D$ entre los grupos estudiados (Tabla 3). De acuerdo con los factores de riesgo analizados, la presencia de alguna enfermedad mostró una razón de momios (RM) de 5.6 (IC95\%, 1.57- 20.17; $p=.005)$; el TTPa registró una RM de 0.30 (IC95\%, 0.12-0.79; $p=0.010$ ). Cuando la variable de "padecimientos previos" se dividió por enfermedad, la RM se diluyó y no se identificó significancia estadística (Tabla 4). No se observó significancia estadística para la edad, peso o género (Tabla 4). Se registraron 28 defunciones en la población estudiada, cinco en los 
casos y 23 en los controles (33.3\%, $n=2$ mujeres $/ n$ $=3$ hombres para los casos), con mortalidad más elevada en la trombosis venosa. La RM para mortalidad en pacientes con episodio trombótico fue de 0.41 (IC95\%, 0.12-1.4; $p=0.15$ ), la tasa de mortalidad en la población con trombosis fue de 178 por cada 1,000 en comparación con 821 por cada 1,000.

\section{Discusión}

Las vasculares son complicaciones graves que pueden aparecer en los pacientes afectados por la COVID-19. Estas complicaciones, provocadas por la activación desmedida del sistema inmunitario al tratar de destruir al virus SARS-CoV-2, pueden ser graves y requerir un tratamiento farmacológico $\mathrm{o}$, en algunos casos, una intervención quirúrgica. Cada vez hay más pruebas que demuestran que los pacientes con COVID-19 son propensos a sufrir complicaciones trombóticas $^{3,9,10}$. El $13.3 \%$ de esta serie no tenía datos respiratorios activos, con pruebas ya negativas pero con antecedente de positividad, lo que sugiere que el riesgo de hipercoagulabilidad de COVID-19 podría no correlacionarse con el grado de respuesta inflamatoria ${ }^{18}$. La evidencia creciente ha demostrado endotelitis inducida por el virus SARS-CoV-2, que también podría contribuir a la hipercoagulación ${ }^{4,10}$. La mayoría de estos pacientes (73.3\%) presentaba al menos alguna comorbilidad adicional, lo cual representa uno de los factores predisponentes para inducir un proceso trombótico en algún momento, riesgo que se eleva al tener más de dos comorbilidades. Se ha demostrado que el sexo femenino y la edad avanzada son factores de mal pronóstico después de las intervenciones de cirugía vascular; en la población de estudio, por el contrario, la incidencia de afectación trombótica fue mayor en el sexo masculino, pero se requiere una muestra más grande para determinar de manera adecuada la relación con el género. El sexo masculino puede relacionarse con un mal pronóstico, al menos en este estudio. Tampoco la diferencia de edades representó algo significativo o un factor de riesgo para determinar un proceso inflamatorio a nivel vascular. Numerosos estudios han demostrado que los pacientes con COVID-19 muestran múltiples anomalías de laboratorio, como valores elevados del dímero D, tiempo de tromboplastina parcial reducido, productos de degradación de fibrina elevados y aumento del fibrinógeno ${ }^{10,13}$. En los pacientes del estudio, algunos valores de laboratorio estaban marcadamente elevados, incluidos el dímero $D(p=0.41)$, la lactato deshidrogenasa y un consumo de plaquetas en el recuento; en cambio, a diferencia de lo establecido en las publicaciones médicas, el tiempo de tromboplastina fue elevado $(p=0.46)$.

El umbral para indicar una intervención quirúrgica depende del estado respiratorio del paciente, la gravedad de la isquemia y el pronóstico general. Se realizaron en este estudio al menos cinco amputaciones debido a diagnósticos tardíos, prioridad sobre enfermedad respiratoria o tan solo por pobre identificación de casos por parte de otros médicos de contacto primario, así como dificultad para realizar la valoración integral que conlleva el acercamiento a estos pacientes durante la hospitalización. El índice elevado de amputaciones se debió a que la afección vascular había pasado a segundo término por la enfermedad respiratoria o los pacientes acudieron de modo tardío a hospitalización por temor y, en consecuencia, cuando se llevó a cabo la valoración los pacientes ya tenían isquemia irreversible. El resto de los tratamientos quirúrgicos se practicó con éxito sin complicaciones, con adecuados flujos posteriores a la revascularización, incluso con uso de un injerto protésico en un caso que demostró permeabilidad adecuada al final, pese a un estado inflamatorio continuo. Por lo tanto, aunque los episodios trombóticos arteriales agudos son un marcador de mal pronóstico para las complicaciones y la mortalidad, las tasas de permeabilidad a corto plazo y de recuperación de la extremidad se mantuvieron altas. Las trombosis venosas se trataron en su totalidad con atención médica y medidas de elastocompresión, si bien la mortalidad fue más elevada.

Este estudio tuvo varias limitaciones. Primero, el número de episodios trombóticos agudos en la institución fue probablemente menor que el notificado en otras series. Esto podría deberse a resultados de pruebas falsas negativas con hisopos nasales, así como a la identificación tardía, selección de diagnósticos diferenciales, evolución tórpida o muerte prematura, o identificación oportuna pero con atención de médicos de atención primaria en esta contingencia, sobre todo para los episodios venosos. Otra de las debilidades del estudio es que no es posible identificar otro tipo de marcadores por la escasez de reactivos, cantidad de pacientes y recursos limitados. Se necesitan más estudios con mayor número de datos y tal vez se puedan usar cifras elevadas del dímero $D$ como marcadores para reconocer a pacientes con un alto riesgo de complicaciones trombóticas por COVID-19.

Las presentaciones vasculares agudas de COVID-19 han dado lugar a varias consideraciones singulares e importantes medidas de tratamiento ${ }^{17}$. Primero, todos los pacientes durante la pandemia que presenten episodios trombóticos deben someterse a la evidencia de 
COVID-19 porque su presencia podría ser un factor de riesgo importante. El aumento de las tasas de episodios trombóticos también enfatiza la relevancia de contra con un cirujano vascular durante la pandemia, al menos en los grandes centros, así como iniciar de manera oportuna la anticoagulación profiláctica y mantenerla cuando menos durante el egreso en pacientes con elevación de algún marcador bioquímico o se considere de alto riesgo para sufrir trombosis ${ }^{1,2}$. Por último, la incidencia de episodios trombóticos en este estudio pudo subregistrarse porque muchos pacientes optaron por quedarse en casa y no presentarse hasta después del período de estudio, tratarse en otro centro o se los valoró de manera errónea.

\section{Conclusiones}

Los pacientes con infección por COVID-19 tienen hipercoagulabilidad, lo que les confiere un alto riesgo de sufrir tromboembolismo arterial y venoso. Para un tamizaje importante en el resto de pacientes, la elevación significativa del dímero $D$ puede ser uno de los marcadores de inflamación más significativo, así como la prolongación del tiempo de tromboplastina. La presentación de comorbilidades como diabetes mellitus e hipertensión arterial sistémica confirma que son factores exponenciales trombóticos en estos pacientes, lo que suscita el interés por el significado de las enfermedades crónicas degenerativas en esta pandemia. En la mayoría de los pacientes fue posible establecer tratamientos médicos y procedimientos de revascularización, con escasos datos de complicaciones y resultados adecuados, incluida una derivación con injerto protésico. Contraer la COVID-19 no es contraindicación para practicar un tratamiento quirúrgico de revascularización, lo que debe llevar a mejorar la conducta diagnóstica y terapéutica en pacientes con infección de COVID-19, así como a buscar identificadores clínicos y bioquímicos para detectar episodios trombóticos con oportunidad y prevenirlos.

\section{Financiamiento}

El equipo y el material son propiedad del Instituto Mexicano del Seguro Social (IMSS). No se contó con financiamiento distinto del de la institución.

\section{Conflicto de intereses}

Los autores declaran no tener conflicto alguno de intereses.

\section{Responsabilidades éticas}

Protección de personas y animales. Los autores declaran que para esta investigación no se han realizado experimentos en seres humanos ni en animales.

Confidencialidad de los datos. Los autores declaran que han seguido los protocolos de su centro de trabajo sobre la publicación de datos de pacientes.

Derecho a la privacidad y consentimiento informado. Los autores han obtenido el consentimiento informado de los pacientes o sujetos referidos en el artículo.

\section{Bibliografía}

1. Bikdeli B, Madhavan MV, Jimenez D. COVID-19 y enfermedad trombótica o tromboembólica: implicaciones para la prevención, la terapia antitrombótica y el seguimiento: revisión del estado del arte de JACC. J Am Coll Cardiol. 2020 Jun 16;75(23):2950-73.

2. Baeza C, González A, Torres P. Acute aortic thrombosis in COVID-19. J Vasc Surg Cases Innov Tech. 2020 Sep;6(3):483-6.

3. McGonagle D, Sharif K, O'Regan A, Bridgewood C. The role of cytokines including interleukin-6 in COVID-19 induced pneumonia and macrophage activation syndrome-like disease. Autoimmun Rev. 2020;19:10253.

4. Cheruiyot I, Kipkorir V, Ngure B. Arterial thrombosis in coronavirus disease 2019 patients: a rapid systematic review. Ann Vasc Surg. 2020 Aug 28;(20):1-9.

5. Henry BM, Vikse J, Benoit S. Hyperinflammation and derangement of renin-angiotensin-aldosterone system in COVID-19: a novel hypothesis for clinically suspected hypercoagulopathy and microvascular immunothrombosis. Clin Chim Acta. 2020 Aug;507:167-73.

6. Kaur P, Qaqa F, Ramahi A. Acute upper limb ischemia in a patient with COVID-19. Hematol Oncol Stem Cell Ther. 2020 May 13:1-3.

7. Zhang H, Penninger JM, Li Y. Angiotensin-converting enzyme 2 (ACE2) as a SARS-CoV-2 receptor: molecular mechanisms and potential therapeutic target. Intensive Care Med. 2020 Apr;46(4):586-90.

8. Zhou F, Yu T, Du R. Clinical course and risk factors for mortality of adult inpatients with COVID-19 in Wuhan, China: a retrospective cohort study. Lancet. 2020 Mar 28;395(10229):1054-62.

9. Spiezia L, Boscolo A, Poletto F. COVID-19-related severe hypercoagulability in patients admitted to intensive care unit for acute respiratory failure. Thromb Haemost. 2020 Jun;120(6):998-1000.

10. Tang N, Li D, Wang X. Abnormal coagulation parameters are associated with poor prognosis in patients with novel coronavirus pneumonia. $J$ Thromb Haemost. 2020 Apr;18(4):844-7.

11. Vlot EA, Van den Dool EJ, Hackeng CM. Anti Xa activity after high dose LMWH thrombosis prophylaxis in COVID-19 patients at the intensive care unit. Thromb Res. 2020 Jul 22;196:1-3.

12. Campbell $\mathrm{CM}$, Kahwash R. Will complement inhibition be the new target in treating COVID-19-related systemic thrombosis? Circulation. 2020 Jun 2;141(22):1739-1741.

13. Varga Z, Flammer AJ, Steiger P. Endothelial cell infection and endotheliitis in COVID-19. Lancet. 2020 May 2;395(10234):1417-8.

14. Bellosta R, Luzzani L, Natalini G. Acute limb ischemia in patients with COVID-19 pneumonia. J Vasc Surg. 2020 Apr 29: 31080-6.

15. Man JJ, Beckman JA, Jaffe IZ. Sex as a biological variable in atherosclerosis. Circ Res. 2020 Apr 24;126(9):1297-1319.

16. Boukhris M, Hillani A, Moroni F. Cardiovascular implications of the COVID-19 pandemic: a global perspective. Can J Cardiol. 2020 Jul;36(7):1068-80.

17. Ilonzo N, Rao A, Safir S. Acute thrombotic manifestations of covid-19 infection: experience at a large New York City health system. J Vasc Surg. 2020 Aug 31:1-18.

18. Klok FA, Kruip MJHA, van der Meer NJM. Confirmation of the high cumulative incidence of thrombotic complications in critically ill ICU patients with COVID-19: an updated analysis. Thromb Res. 2020 Jul;191:148-150.

19. Klok FA, Kruip MJHA, van der Meer NJM. Incidence of thrombotic complications in critically ill ICU patients with COVID-19. Thromb Res. 2020 Jul;191:145-147.

20. Vacirca A, Faggioli G, Pini R. Unheralded lower limb threatening ischemia in a COVID-19 patient. Int J Infect Dis. $2020 \mathrm{Jul} ; 96: 590-2$.

21. Gasmi A, Massimiliano P, Pivina L. Interrelations between COVID-19 and other disorders. Clinical Inmunology. 2021 Mar;224:108651. 